\title{
Adaptive technique for phase noise reduction from the speckle correlation fringes
}

\author{
173 \\ Phanindra Narayan Gundu*a, Erwin Hack ${ }^{\mathrm{a}}$, Pramod K. Rastogi ${ }^{\mathrm{b}}$ \\ ${ }^{a}$ Electronics/Metrology Laboratory, Swiss Federal Laboratories for Materials Testing and Research \\ (EMPA), Ueberlandstrasse 129, CH-8600 Duebendorf, Switzerland; \\ ${ }^{\mathrm{b}}$ Applied Computing and Mechanics Laboratory (IMAC), Swiss Federal Institute of Technology \\ Lausanne, CH-1015 Lausanne, Switzerland
}

\begin{abstract}
We show improvement in the signal-to-noise ratio of speckle correlation fringe patterns by providing a conjugate phase to the scattered object beam. This conjugate phase negates the speckle phase and will leave the speckle intensity as the only statistically varying quantity. Since one of the random variables is reduced the signal-to-noise ratio improves. The variation of intensity in the fringe profile is reduced after the adaptive phase correction. This improvement is vividly seen after a simple morphological filtering. The experiment is performed using a twisted nematic liquid crystal display SLM in transmission.
\end{abstract}

Keywords: Adaptive optics, ESPI, speckle phase, noise reduction, filtering, LCD, SLM.

\section{INTRODUCTION}

The fact that light can be modulated using Spatial Light Modulators (SLMs) has opened many possible applications in traditional optics. In ESPI, deformations and displacements on optically rough surfaces are measured. To evaluate the deformation or displacement, several interferograms are captured with a change in the phase of one of the interfering beams to evaluate the initial surface profile or phase distribution. The same method is again performed to find the final phase distribution. From the difference of these two states the deformation is evaluated. There are several phase stepping algorithms which differ in the amount of phase step angle and the number of phase steps ${ }^{1,2}$. This process is not only time consuming but also not practicable in the industrial environment. A two step correlation fringe method which involves only subtraction of intensity values of the two interferograms of initial and final states is more apt for industrial environment but contains a high degree of speckle noise in the interference fringes.

Several post processing methods to improve the fringe quality have been reported previously ${ }^{3,4}$, but their success was limited since the signal to noise ratio for speckle noise is near to 1 . The treatment of speckle noise using image processing techniques results in blurring or reduction of contrast, and the phase function evaluated has errors. As one can observe from the mathematical treatment of the speckle fringe pattern ${ }^{5,6}$, the noise in the speckle fringes is due to both the random phase and amplitude of the speckle field scattered from the object.

In this article, we first show by simulation how the $\mathrm{S} / \mathrm{N}$ ratio of the correlation fringe pattern is improved by providing a conjugate phase to the scattered object beam. This conjugate phase will negate the speckle phase and will leave the speckle intensity as the only statistically varying quantity. Since one of the random variables is reduced the $\mathrm{S} / \mathrm{N}$ ratio improves. The variation of intensity in the fringe profile is reduced after the adaptive phase correction. This improving is vividly seen after a simple morphological filtering.

The experiment is performed using a twisted nematic liquid crystal display SLM in transmission. Initially, this LCD is characterized to perform as phase-only SLM. Then the LCD SLM is placed in the speckle interferometer to suppress the speckle phase. Later, filtering is done to the corrected fringe pattern.

* phanindra.gundualempa.ch; phone 4144823 4863; fax 4144823 4054; www.empa.ch 


\section{THEORY}

The deformation of an object results in speckle pattern correlation fringes from the subtraction of interferograms. These interferograms are taken when the object is undeformed $I_{C C D, \text { initial }}$ and deformed $I_{C C D \text { final }}$ states. The intensity difference can be written as ${ }^{6}$ :

$$
\left|\mathrm{I}_{\mathrm{CCD}, \text { final }}-\mathrm{I}_{\mathrm{CCD} \text {,initial }}\right|=2 \mathrm{I}_{\mathrm{M}} \mid \sin \left(\phi_{\mathrm{S}}-\phi_{\mathrm{R}}+\frac{\Delta \phi}{2}\right) \sin \left(\frac{\Delta \phi}{2}\right)
$$

where $I_{M}$ is the modulation intensity which is the product of square root of the speckle and reference intensities, $\phi_{S}$ is the speckle phase, $\phi_{\mathrm{R}}$ is the reference phase and $\Delta \phi$ is the phase difference due to deformation. Eq.(1) can be rewritten in short form as $\mathrm{I}_{\text {diff. }}=\mathrm{I}_{\mathrm{M}} \mathrm{P}_{\mathrm{S}} \mathrm{D}$; where $P_{S} \equiv \mid \sin \left(\phi_{\mathrm{S}}-\phi_{\mathrm{R}}+\frac{\Delta \phi}{2}\right)$ and $\mathrm{D} \equiv 2 \mid \sin \left(\frac{\Delta \phi}{2}\right)$. The first two terms are varying statistically because of intensity and phase distribution of the speckle field, respectively. Reduction of noise in the speckle pattern can be achieved by reducing these statistically varying quantities. Reducing the width of the probability density function of at least one of the quantities will increase the fringe quality ${ }^{7}$. The average and variance of $I_{\text {diff }}$ for a region with constant phase difference $D_{0}$ can be written as

$$
<\mathrm{I}_{\text {diff }}>=<\mathrm{I}_{\mathrm{M}} \mathrm{P}_{\mathrm{S}}>\mathrm{D}_{\mathrm{O}}
$$

and

$$
\operatorname{var}\left[\mathrm{I}_{\mathrm{diff}}\right]=<\mathrm{I}_{\mathrm{M}}^{2} \mathrm{P}_{\mathrm{S}}^{2}>\mathrm{D}_{\mathrm{O}}{ }^{2}-<\mathrm{I}_{\mathrm{M}} \mathrm{P}_{\mathrm{S}}>^{2} \mathrm{D}_{\mathrm{O}}^{2}
$$

respectively. Because $I_{M}$ and $P_{S}$ are statistically independent, the signal to noise ratio can be written as:

$$
S N R_{u n c}^{2}=\frac{\left\langle I_{\text {diff }}\right\rangle^{2}}{\operatorname{var}\left[\mathrm{I}_{\text {diff }}\right]}=\frac{\left\langle I_{M}\right\rangle^{2}\left\langle P_{S}\right\rangle^{2}}{\left\langle I_{M}^{2}\right\rangle\left\langle P_{S}{ }^{2}\right\rangle-\left\langle I_{M}\right\rangle^{2}\left\langle P_{S}\right\rangle^{2}}
$$

where the subscript 'unc' identifies the uncorrected fringe pattern. Substituting the variances of speckle phase $\left(\operatorname{var}\left[\mathrm{P}_{\mathrm{S}}\right]=\right.$ $\left.<\mathrm{P}_{\mathrm{S}}{ }^{2}>-<\mathrm{P}_{\mathrm{S}}>^{2}\right)$ and modulation intensity $\left.\left(\operatorname{var}\left[\mathrm{I}_{\mathrm{M}}\right]=\left\langle\mathrm{I}_{\mathrm{M}}{ }^{2}\right\rangle-<\mathrm{I}_{\mathrm{M}}\right\rangle^{2}\right)$ in Eq. (4), we get

$$
S N R_{u n c}^{2}=\frac{\left\langle I_{M}\right\rangle^{2}\left\langle P_{S}\right\rangle^{2}}{\left\langle I_{M}^{2}\right\rangle \operatorname{var}\left[P_{S}\right]+\left\langle P_{S}\right\rangle^{2} \operatorname{var}\left[I_{M}\right]}
$$

If the speckle phase is made uniform by an adaptive method, then $\left\langle\mathrm{P}_{\mathrm{S}}{ }^{2}\right\rangle=\left\langle\mathrm{P}_{\mathrm{S}}\right\rangle^{2}$. Substituting this in Eq.(4) we get the $\mathrm{SNR}$ for the corrected fringes, $\mathrm{SNR}_{\text {cor }}$. The gain in $\mathrm{S} / \mathrm{N}$ ratio is given by the ratio of the two SNRs:

$$
\Gamma=\frac{S N R_{\text {cor }}^{2}}{S N R_{\text {unc }}^{2}}=\frac{\left\langle I_{M}\right\rangle^{2}\left[\left\langle I_{M}^{2}\right\rangle \operatorname{var}\left[P_{S}\right]+\left\langle P_{S}\right\rangle^{2} \operatorname{var}\left[I_{M}\right]\right]}{\operatorname{var}\left[I_{M}\right\}\left\langle I_{M}\right\rangle^{2}\left\langle P_{S}\right\rangle^{2}}=1+\frac{\operatorname{var}\left[P_{S}\right\}\left\langle I_{M}\right\rangle^{2}}{\left\langle P_{S}\right\rangle^{2} \operatorname{var}\left[I_{M}\right]}>1
$$


Eq.(6) shows that the SNR improves as one of the two terms in the speckle noise is corrected.

\section{SIMULATION}

A speckle pattern is generated using the statistics of speckle phase and amplitude. Here the average number of speckles per pixel is chosen 1 . This speckle pattern is added interferometrically to a plane reference wave. A wedge shape deformation (tilt) is simulated to obtain a second interferogram. These speckle interferograms are subtracted pixel-wise to obtain the correlation fringe pattern (Figure 1). A line intensity profile of this fringe pattern along a row is also shown. Fig. 2 shows the fringe pattern after adaptive speckle phase correction with the conjugate wavefront. It can be observed from the line intensity profile of fig. 2 that this adaptive correction rectifies the intensity pattern to produce a clearly visible envelope interspersed with gaps. The filling of these gaps which is identical to connecting the disjoint groups of intensity objects can be performed using a filtering method called dilation in morphology ${ }^{8}$. The morphological dilation operation with the gray scale image is performed with a structuring element. When the structuring element is translated over the image, the maximum value lying within the structuring element is chosen to replace the common center pixel value. A 3 X3 cross structuring element is chosen to filter Figure 2. Figure 3 shows the resultant fringe pattern after phase correction and dilation operation. In this figure all the gaps are filled and the fringe envelope is preserved which is not the case with traditional filtering operations.
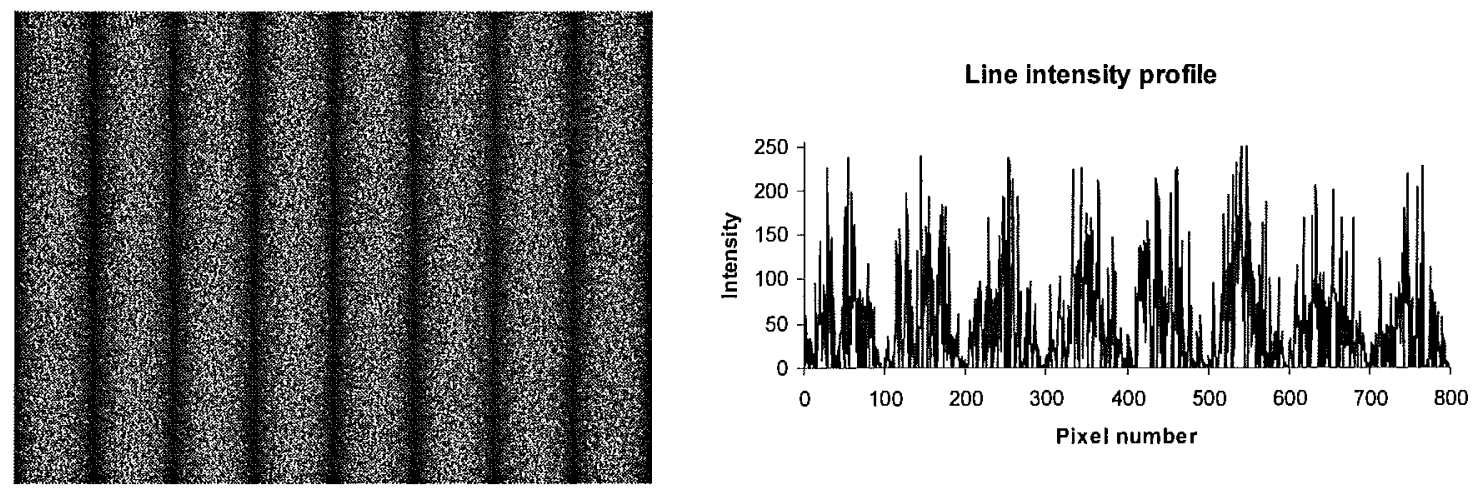

Figure 1: Speckle correlation fringe pattern and its line intensity profile.
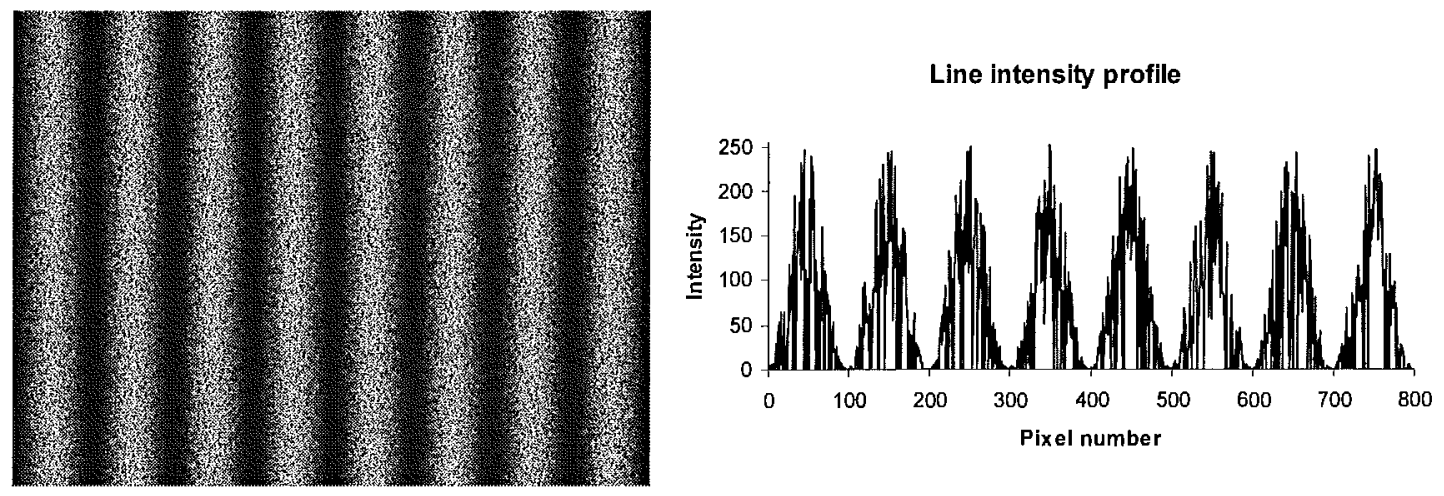

Figure 2: Speckle correlation fringe pattern after adaptive correction and its line intensity profile. 

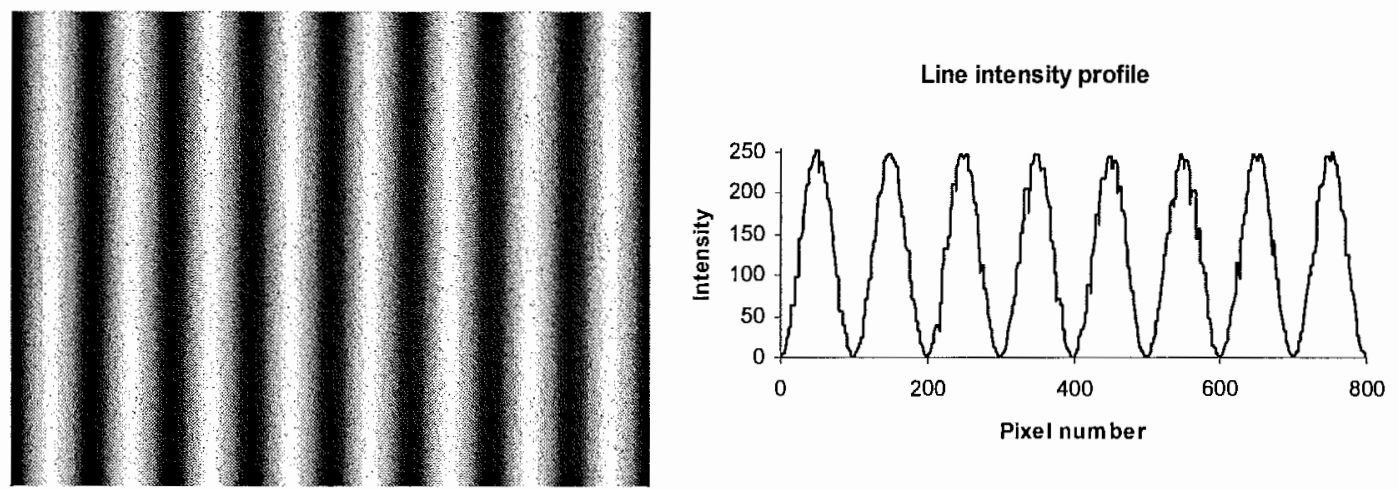

Figure 3: Speckle correlation fringe pattern after dilation operation of fig. 2 and its line intensity profile.

\section{EXPERIMENT}

The experiment is performed using a twisted nematic LCD SLM. We use a high spatial resolution (SVGA) LCD, the Sony LCX016AL having $832 \times 624$ pixels with a pixel pitch of $32 \mu \mathrm{m}$ and 1.3 inch diagonal size. To prepare the LCD to work as phase-only SLM, it is sandwiched between two quarter wave plates and linear polarizers. The characterization of the LCD SLM is performed using a Mach-Zehnder interferometer. The method of characterization is widely explained in Refs. 9 and 10. The variation of phase and intensity of this phase-only LCD SLM is shown in Figure 4 . The variation of phase is from 0 to 214 degrees, while the intensity variation is only about $\pm 5 \%$ over the entire range which can be seen from figure 4.

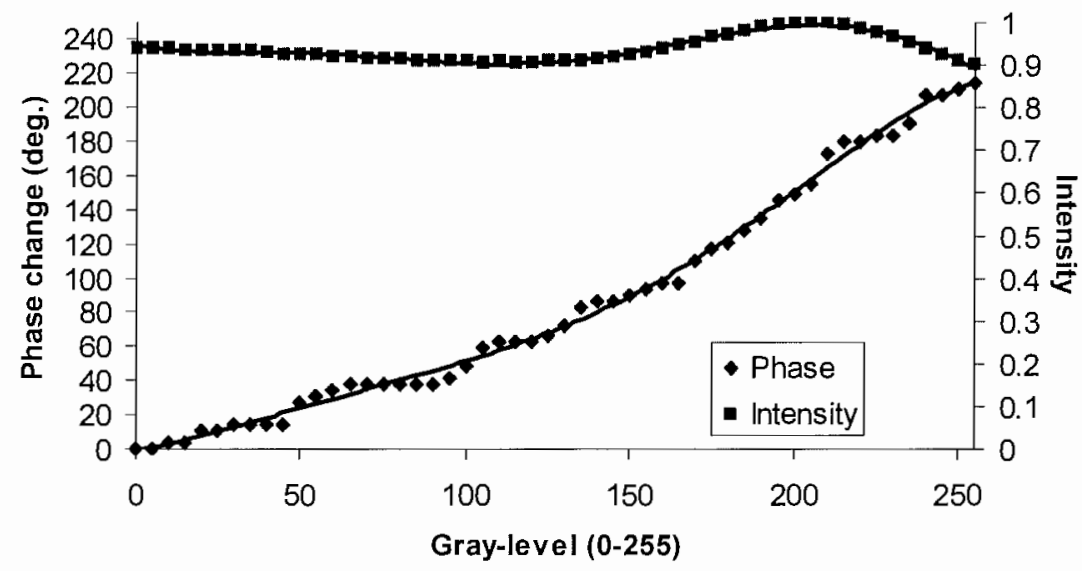

Figure 4: The characteristic graph of phase and intensity change of the LCD SLM.

The ESPI setup for speckle phase correction is shown in figure 5. The LCD SLM is inserted in one of the arms of the interferometer. The beam scattered from the object and the beam transmitted through the LCD are imaged using L2 and L3, respectively. Here the lens positions are adjusted to the proper magnifications, i.e. to accommodate the area of interest on the object and to match the $32 \mu \mathrm{m}$ pixels of the LCD to the $11 \mu \mathrm{m}$ pixel pitch of the CCD.

The maximum phase change of $214 \mathrm{deg}$ is sufficient to correct the speckle phases.since from Eq. (1) we need only the modulus of the sine-function for which $\left|\sin \left(\phi_{\mathrm{S}}-\phi_{\mathrm{R}}+\frac{1}{2} \Delta \phi\right)\right|=\mid \sin \left(\phi_{\mathrm{S}}-\phi_{\mathrm{R}}+\pi+\frac{1}{2} \Delta \phi \mid\right.$. 
In order to obtain the speckle phase, the phase-only LCD itself is used as the phase shifter by giving a constant gray value to all the pixels in order to obtain a constant phase step. A three frame phase stepping method is chosen and gray values of 0,150 and 220 are evaluated for phase changes of $0, \pi / 2$ and $\pi$ based on the phase change characteristic graph in Fig. 4. The speckle phase information is fed back to the LCD in order to cancel the phase difference $\left(\phi_{\mathrm{S}}-\phi_{\mathrm{R}}\right)$ in the sine function in Eq. 1, from which

$$
\left|I_{C C D, \text { final }}-I_{C C D, \text { initial }}\right|^{\text {corrected }}=2 I_{M} \mid \sin ^{2}\left(\frac{\Delta \phi}{2}\right)
$$

Figures $6(\mathrm{~A})$ and $6(\mathrm{~B})$ are the fringe patterns without and with adaptive phase correction obtained after a tilt of the rough object in the setup shown in figure 5 . The fourth fringe shown by the arrow is more clearly seen in the fringe pattern with adaptive correction. The streaks in between second and the third fringes which developed due to the multiple reflections between the optical elements are absent in figure $6(\mathrm{~B})$. Overall the contrast of the fringe pattern after adaptive correction is higher.

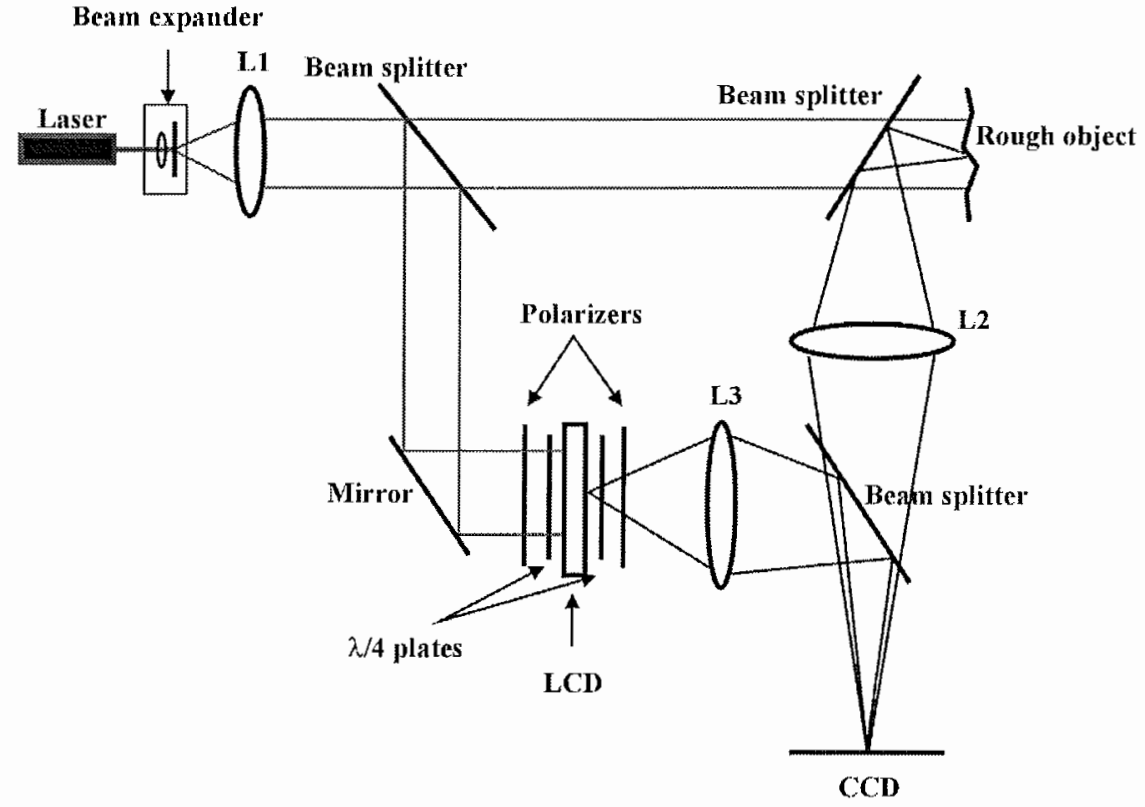

Figure 5: Adaptive ESPI setup 


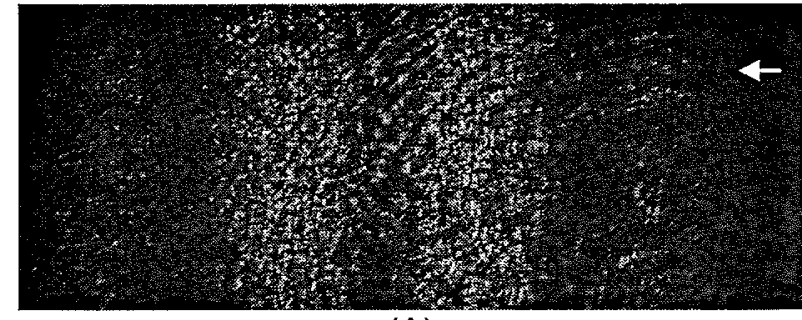

(A)

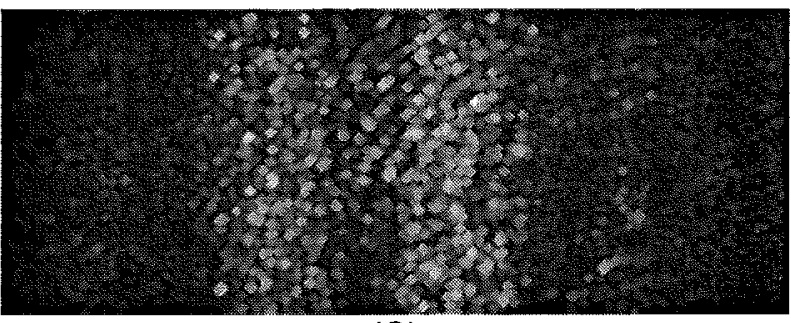

(C)

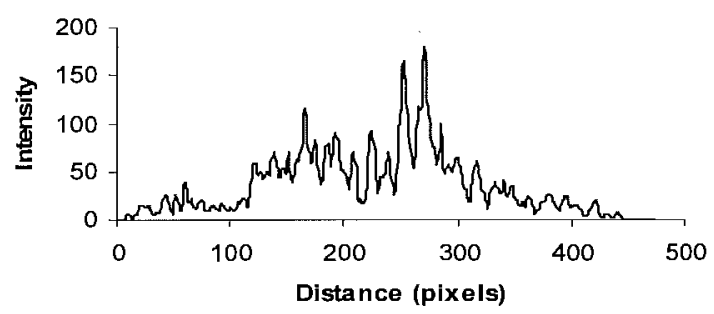

(E)

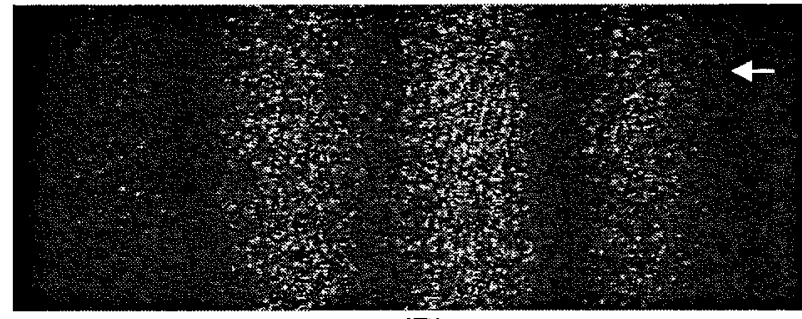

(B)

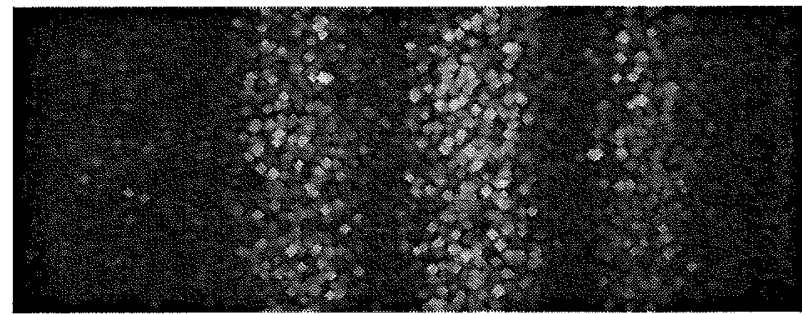

(D)

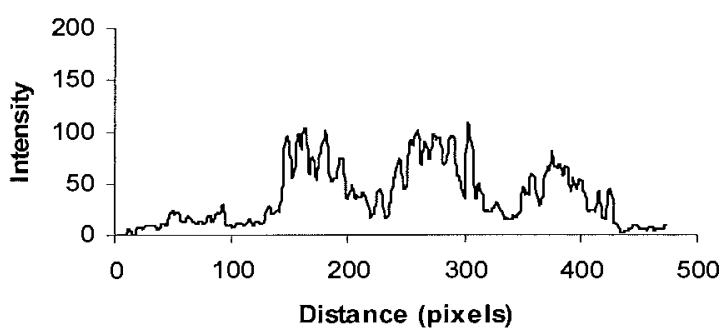

(F)

Figure 6: (A) and (B) are the speckle correlation fringes without and with adaptive correction, (C) and (D) are the respective fringe patterns after dilation operation with a $3 \times 3$ cross element, $(E)$ and $(F)$ represent the line intensity profiles of (C) and (D) along the rows.

\section{CONCLUSION}

We have shown by theory, simulation and experiment an improvement in the speckle correlation fringes due to adaptive phase correction. Since the phase only SLM has no smooth graylevel-phase-characteristics, the achieved correction was only partial which can be observed from the line intensity profiles where many gaps still exist. However, the contrast improvement produced by the SLM is clearly seen.

\section{REFERENCES}

1. Y. Surrel, "Design of algorithms for phase measurements by the use of phase stepping", Appl. Opt. 35, 51-60 (1996).

2. A. Patil, P. Rastogi and R. Langoju, "High-resolution frequency estimation technique for recovering phase distribution in interferometers," Opt. Lett. 30, 391-393 (2005). 
3. J.S. Lim, H. Nawab, "Techniques for speckle noise removal", Proc. of SPIE 243, 35-44 (1980).

4. A. Federico, G.H. Kaufmann, "Comparative study of wavelet thresholding methods for denoising electronic speckle pattern Interferometry fringes”, Opt. Eng. 40, 2598-2604 (2001).

5. P.K. Rastogi (Ed.), Digital speckle pattern interferometry and related techniques (John Wiley \& Sons Ltd, Chichester, England, 2001).

6. E. Hack, "ESPI - Principles and prospects", in Trends in optical non-destructive testing and inspection (Elsevier Science Ltd., Oxford, UK), 207-239 (2000).

7. E. Hack, P.N. Gundu and P. Rastogi, "Adaptive correction to the speckle correlation fringes by using a twistednematic liquid-crystal display,” Appl. Opt. 44, 2772-2781(2005).

8. R.C. Gonzalez and R.E. Woods, Digital Image Processing (Addison-Wesley publishing company Inc., Massachusetts, 1993).

9. J.L. Pezzaniti and R.A. Chipaman, "Phase-only modulation of a twisted nematic liquid-crystal TV by use of the eigenpolarization states", Opt. Lett. 18, 1567-1569 (1993).

10. J.A. Davis, I. Moreno, and P. Tsai, "Polarization eigenstates for twisted-nematic liquid crstal displays", Appl. Opt. 37, 937-945 (1998). 


\section{Volume 5908 -- Optical Information Systems III}

Bahram Javidi, Demetri Psaltis, Editors, September 2005

Conference Location: San Diego, CA, USA

Conference Date: 3 August 2005 